Cite this: Phys. Chem. Chem. Phys. 2014, 16, 12812

Received 26th March 2014 Accepted 29th April 2014

DOI: $10.1039 / c 4 c p 01306 a$

www.rsc.org/pccp

\section{Dynamic control of Förster energy transfer in a photonic environment}

\begin{abstract}
Frank Schleifenbaum, Andreas M. Kern, Alexander Konrad* and Alfred J. Meixner*
In this study, the effect of modified optical density of states on the rate of Förster resonant energy transfer between two closely-spaced chromophores is investigated. A model based on a system of coupled rate equations is derived to predict the influence of the environment on the molecular system. Due to the near-field character of Förster transfer, the corresponding rate constant is shown to be nearly independent of the optical mode density. An optical resonator can, however, effectively modify the donor and acceptor populations, leading to a dramatic change in the Förster transfer rate. Single-molecule measurements on the autofluorescent protein DsRed using a $\lambda / 2$-microresonator are presented and compared to the theoretical model's predictions. The observed resonator-induced dequenching of the donor subunit in DsRed is accurately reproduced by the model, allowing a direct measurement of the Förster transfer rate in this otherwise inseparable multichromophoric system. With this accurate yet simple theoretical framework, new experiments can be conceived to measure normally obscured energy transfer channels in complex coupled quantum systems, e.g. in photovoltaics or light harvesting complexes.
\end{abstract}

\section{Introduction}

Since the early work of Förster, ${ }^{1}$ the interaction between closely spaced fluorescent molecules via dipole-dipole coupling (fluorescence/Förster resonant energy transfer, FRET) has been a topic of great interest. ${ }^{2-9}$ The efficiency of the energy transfer depends on the spectral overlap between the emission of the donor chromophore and the absorption of the acceptor chromophore as well as on the distance and the mutual orientation of their respective transition dipole moments. While it is easily possible to design and prepare synthetic FRET-pairs and study the optical properties of the individual chromophores separately, this is not possible for many biological molecules such as the red fluorescent protein DsRed from the Discosoma reef coral. DsRed is known to form tetramers consisting of two different types of subunits containing a chromophore exhibiting fluorescence emission either in the green or red spectral regime. ${ }^{10-12}$ These spectral properties along with the steric composition as derived from X-ray data suggest a non-radiative Förster energy transfer within a tetrameric unit which has indeed been experimentally proven by different spectroscopic approaches using single molecule and ensemble techniques. ${ }^{2,11,13,14}$ However, it is not possible to separate the tetramers into functional monomers by chemical or biochemical means to make the isolated chromophoric species addressable for further investigation.

Universität Tübingen, Institut für Physikalische und Theoretische Chemie, Auf der Morgenstelle 18, 72076 Tübingen, Germany. E-mail: x@uni-tuebingen.de; Fax: +49-7071-29-5490; Tel: +49-7071-29-76
A promising approach to spectrally isolate individual chromophoric subunits in biological FRET-systems is to modify the local photonic mode characteristics and density by using a $\lambda / 2$-microresonator. We have previously demonstrated the optical confinement effect on both the fluorescence spectrum and the emission rate of single (synthetic) dye molecules by embedding them in a transparent polymer between two planar silver mirrors separated by half of the emission wavelength. ${ }^{15,16}$ The influence of the modified photonic mode density on a system consisting of coupled molecular dipole emitters is theoretically well described ${ }^{17-22}$ and several experimental demonstrations on the ensemble level have been reported to date..$^{23-26}$

In this article, we report the first investigation of the autofluorescent protein DsRed embedded in a $\lambda / 2$-microresonator by steady-state and time resolved spectroscopy down to the single molecule level. We use a novel microresonator design that allows coupling the fluorescence of individual chromophores to on- and off-axis cavity modes while maintaining physiological conditions for the embedded biomolecules. We show that, in this way, it is possible to spectrally isolate the two coupled chromophoric subunits of DsRed without destroying the composition of the tetrameric protein complex.

\section{Rate equation model}

To study the effect of a photonic environment on a FRETcoupled system, we introduce a rate equation model describing the energetic processes of the system. Shown in Fig. 1, this 
model comprises two three-level subsystems D and A representing the donor and acceptor molecules, respectively. Each subsystem $\mathrm{X}$ $(\mathrm{X}=\mathrm{D}, \mathrm{A})$ can be excited at the rate $X_{0} k_{\text {exc }}=X_{0} J \sigma$, where $X_{0}$ is the probability to find the subsystem in its electronic ground state, $k_{\text {exc }}$ is the excitation rate constant, $J$ is the incident illumination photon flux at the absorption wavelength and $\sigma$ is the corresponding absorption cross section. Optical excitation of $X_{0}$ leads to a vibronic level in the first electronically excited state $\mathrm{X}_{1}{ }^{\prime}$ which thermally relaxes rapidly within some picoseconds to $X_{1}$, from which it may decay nonradiatively or radiatively at the rates $X_{1} k_{\mathrm{nr}}$ and $X_{1} k_{\mathrm{rad}}$, respectively. Here, $k_{\mathrm{nr}}$ and $k_{\mathrm{rad}}$ are the nonradiative and radiative decay rate constants, respectively. In addition, the subsystems D and A are coupled via the nonradiative channel representing Förster resonant energy transfer, described by the rate constant $k_{\mathrm{T}}$ as a measure of the dipole-dipole coupling strength. This Förster transition rate is defined as the probability per time interval that an acceptor molecule is transferred from its ground state $A_{0}$ to its electronically excited state $A_{1}$ by absorbing a photon of the optical near-field of the donor chromophore with state $\mathrm{D}_{1}$. The transition rate is then given by $D_{1} A_{0} k_{\mathrm{T}}$.

The population probability dynamics for the excited states $D_{1}$ and $A_{1}$ of the subsystems can be written by a system of coupled differential equations,

$$
\begin{gathered}
\dot{D}_{1}=k_{\mathrm{exc}}^{\mathrm{D}} D_{0}-\left[k_{\mathrm{rad}}^{\mathrm{D}}+k_{\mathrm{nr}}^{\mathrm{D}}+k_{\mathrm{T}} A_{0}\right] D_{1}, \\
\dot{A}_{1}=\left[k_{\mathrm{exc}}^{\mathrm{A}}+k_{\mathrm{T}} D_{1}\right] A_{0}-\left[k_{\mathrm{rad}}^{\mathrm{D}}+k_{\mathrm{nr}}^{\mathrm{D}}+\right] A_{1},
\end{gathered}
$$

where a dotted value denotes a derivative in time. Here, superscripts D and A denote values corresponding to the donor and acceptor subsystems, respectively. In equilibrium, the populations are described by the steady-state solution to eqn (1), obtained for $\dot{D}_{1}=\dot{A}_{1}=0$. With $X_{0}+X_{1}=1$, donor and acceptor excited-state populations can then be written as

$$
\begin{aligned}
D_{1} & =\frac{k_{\mathrm{exc}}^{\mathrm{D}}}{k_{\mathrm{exc}}^{\mathrm{D}}+k_{\mathrm{rad}}^{\mathrm{D}}+k_{\mathrm{nr}}^{\mathrm{D}}+k_{\mathrm{T}}\left(1-A_{1}\right)}, \\
A_{1} & =\frac{k_{\mathrm{exc}}^{\mathrm{A}}+k_{\mathrm{T}} D_{1}}{k_{\mathrm{exc}}^{\mathrm{A}}+k_{\mathrm{rad}}^{\mathrm{A}}+k_{\mathrm{nr}}^{\mathrm{A}}+k_{\mathrm{T}} D_{1}} .
\end{aligned}
$$

When placed in a modified photonic environment, e.g. a resonant cavity, the parameters in eqn (2) can change. First, the intensity of the incident light can be enhanced or suppressed

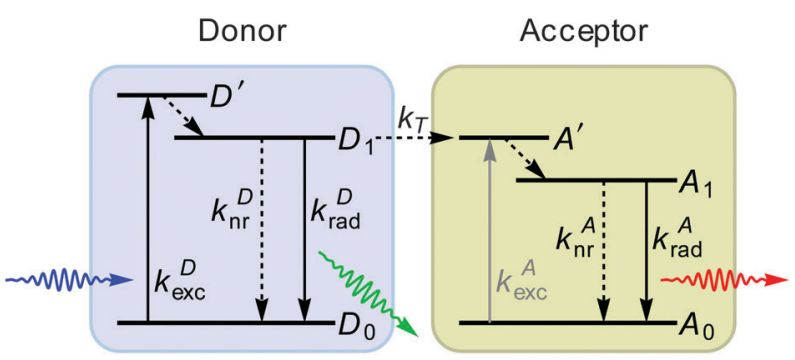

Fig. 1 System used to model the FRET-coupled system. Radiative transitions are shown as solid lines, nonradiative transitions as dashed lines. In our study, the acceptor is not directly excited, hence its excitation channel is drawn in gray. when the cavity is excited on or off resonance, varying the incident photon flux $J$ and thus $k_{\text {exc }}$. With the intensity enhancement factor $F_{\text {exc }}=I(\mathbf{r}) / I_{\mathrm{fs}}(\mathbf{r})$ denoting the incident intensity at the position $\mathbf{r}$ of the quantum system in a photonic environment compared to free space, the modified excitation rate constant can be expressed as $k_{\text {exc }}=F_{\text {exc }} k_{\text {exc,fs }}$.

Second, the radiative decay rate constant $k_{\mathrm{rad}}$ is proportional to the local density of optical states (LDOS) $\rho$ corresponding to the transition energy. In a photonic background, $\rho$ is a function of space and the emitter's orientation and can vary by many orders of magnitude, dramatically changing the behavior of the coupled quantum system. Introducing the LDOS enhancement factor $F_{\text {rad }}=$ $\rho(\mathbf{r}) / \rho_{\mathrm{fs}}(\mathbf{r})$ induced by the photonic environment at the position of the emitter, the radiative decay rate constant can be expressed as $k_{\text {rad }}=$ $F_{\text {rad }} k_{\text {rad,fs. }}$ The value $F_{\text {rad }}$ is also known as the Purcell factor.

Finally, the Förster transfer rate constant $k_{\mathrm{T}}$ can be influenced by the photonic background as well. While FRET is a nonradiative process, often described as a near-field dipole-dipole interaction, it is nevertheless influenced by modifications to the electromagnetic field: if a photonic system enhances the donor dipole's near-field, it will equally enhance the induced dipole moment in the acceptor, thus increasing the FRET speed. The photonic enhancement $F_{\mathrm{T}}=$ $\left|\mathbf{E}^{\mathrm{D}}\left(\mathbf{r}^{\mathrm{A}}\right)\right|^{2} /\left|\mathbf{E}_{\mathrm{fs}}^{\mathrm{D}}\left(\mathbf{r}^{\mathrm{A}}\right)\right|^{2}$ of the donor's dipole field intensity at the position of the acceptor compared to free space thus also describes the enhancement of the FRET channel, $k_{\mathrm{T}}=F_{\mathrm{T}} k_{\mathrm{T}, \mathrm{fs}}$, assuming that there is no change in polarization.

The radiative and FRET enhancement factors can be conveniently computed given the photonic system's electromagnetic response in the form of its dyadic Green's function G. This $3 \times 3$-tensorial function describes the electric field at an arbitrary position $\mathbf{r}^{\prime}$ induced by a single dipole emitter in the photonic system,

$$
\mathbf{E}\left(\mathbf{r}^{\prime}\right)=\omega^{2} \mu(\mathbf{r}) \mathbf{G}\left(\mathbf{r}^{\prime}, \mathbf{r}\right) \cdot \mathbf{p} .
$$

Here, $\mathbf{r}$ is the position of the dipole emitter, $\mathbf{p}$ is its dipole moment, $\hbar \omega$ is the transition energy and $\mu(\mathbf{r})$ is the magnetic permeability at the position of the emitter. The LDOS can directly be computed as

$$
\rho(\mathbf{r})=\frac{6 \pi \omega}{\hbar c^{2}} \operatorname{Im}\left\{G_{\hat{\mathbf{p}}}(\mathbf{r}, \mathbf{r})\right\},
$$

where $G_{\hat{\mathbf{p}}}=\hat{\mathbf{p}} \cdot \mathbf{G} \cdot \hat{\mathbf{p}}$ and $\hat{\mathbf{p}}=\mathbf{p} /|\mathbf{p}|$ is a unit vector in the direction of the emitter's dipole moment. In free space, eqn (4) results analytically in $\rho_{\mathrm{fs}}(\mathbf{r})=\omega^{2} /\left(\hbar c^{3}\right)$; the LDOS is then homogeneous and isotropic. With eqn (4), the radiative enhancement factor can then be written as

$$
F_{\text {rad }}=6 \pi \frac{c}{\omega} \operatorname{Im}\left\{G_{\hat{\mathbf{p}}}(\mathbf{r}, \mathbf{r})\right\} .
$$

The FRET enhancement factor $F_{\mathrm{T}}$ can also be derived from eqn (3) with $\mathbf{r}$ and $\mathbf{r}^{\prime}$ describing the positions of the donor and the acceptor, respectively:

$$
F_{\mathrm{T}}=\frac{\mu\left(\mathbf{r}^{\mathrm{D}}\right)}{\mu_{\mathrm{fs}}\left(\mathbf{r}^{\mathrm{D}}\right)} \frac{\left|\mathbf{G}\left(\mathbf{r}^{\mathrm{A}}, \mathbf{r}^{\mathrm{D}}\right) \cdot \mathbf{p}^{\mathrm{D}}\right|^{2}}{\left|\mathbf{G}_{\mathrm{fs}}\left(\mathbf{r}^{\mathrm{A}}, \mathbf{r}^{\mathrm{D}}\right) \cdot \mathbf{p}^{\mathrm{D}}\right|^{2}} .
$$

The first term in eqn (6) can usually be neglected as the magnetic permeability is seldom changed in a photonic system. 
In the second term, the absolute value of the donor's dipole moment cancels out, and so $F_{\mathrm{T}}$ depends solely on $|\mathbf{G}|^{2}$ in the direction of the donor's dipole moment.

The dyadic Green's function G can be obtained using a number of analytical or numerical approaches. For the simple case of an ideal Fabry-Pérot microresonator, analytical calculations have been presented. ${ }^{27}$ For more complex resonator geometries including multiple layers and interfaces, the transfer matrix method (TMM) provides a quasi-analytical solution. For arbitrary photonic systems, numerical methods such as the finite-difference timedomain (FDTD) ${ }^{28}$ or surface integral equation (SIE) ${ }^{29}$ approach may be required for satisfactory results.

\section{$2.1 \lambda / 2$-Microresonator}

Due to the simple geometry of a $\lambda / 2$-microresonator, its electromagnetic response can be calculated analytically. ${ }^{27}$ The angular dependence of its modes' resonances limits the Purcell factor of a planar Fabry-Pérot-type resonator to at most $F_{\text {rad }}=3$, even for perfectly reflecting mirrors. Emission inhibition, on the other hand, can be very effective, reaching values of nearly $F_{\text {rad }} \approx 0$. The size of a $\lambda / 2$-microresonator is on the order of the emitted light's wavelength, thus only the far field of an embedded emitter can populate its modes: as the near field's amplitude decays with $R^{-3}$, it will have nearly vanished even before reaching the resonator's mirrors for the first time. A comparison of a dipole emitter's far field to its near field shows that the intensity of the far field which is only one wavelength $\lambda$ away is more than 8 orders of magnitude weaker than that of the near field at a distance of $\lambda / 100$. One can thus see that even a large resonant enhancement of the cavity modes will have only a minuscule effect on the FRET rate constant $k_{\mathrm{T}}$.

Changes in the FRET rate $D_{1} A_{0} k_{\mathrm{T}}$ in a $\lambda / 2$-microresonator will therefore not be caused by a change in the rate constant, but instead by the changes in the donor and acceptor populations $D_{1}$ and $A_{0}$, respectively. ${ }^{30}$ In particular, efficient emission inhibition of the donor's emission wavelength can effectively increase its excited state population $D_{1}$, leading to an increase in FRET. Similarly, inhibiting acceptor fluorescence can lead to a depletion of the ground state population $A_{0}$, preventing Förster transfer.

\section{Experimental results}

To observe the effects predicted by our model, we experimentally studied the fluorescence of a Förster-coupled system in a $\lambda / 2$-microresonator. As a FRET system, we chose the autofluorescent protein DsRed, a complex molecule containing two spectrally isolated chromophoric subunits with fluorescence maxima at $505 \mathrm{~nm}$ and $580 \mathrm{~nm}$, respectively. These two subunits can couple nonradiatively, allowing energy to be transferred from the energetically higher subunit to the lower subunit via FRET. The gray shaded area in Fig. 2(b) shows the free-space emission spectrum of DsRed when illuminated at $473 \mathrm{~nm}$, clearly displaying the two fluorescence peaks.

The photonic background in our study was defined by a $\lambda / 2$ Fabry-Pérot microresonator enclosing the DsRed molecules.

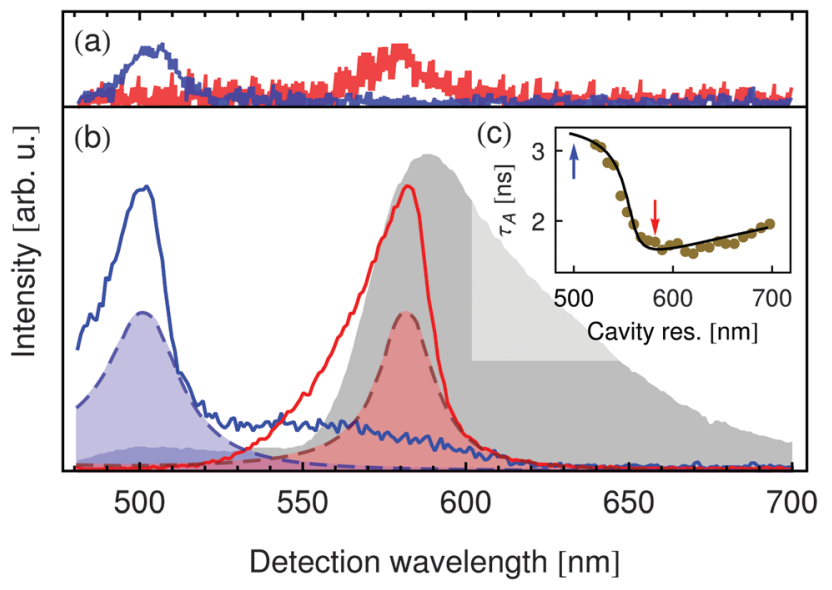

Fig. 2 Measured spectra: (a) single DsRed tetramers in a $\lambda / 2$-microresonator tuned to the donor emission wavelength (blue curve) and acceptor emission (red curve). (b) Ensemble DsRed in free space (gray shaded area) and in $\lambda / 2$-microresonator tuned to the donor emission wavelength (blue curve) and acceptor emission (red curve), along with the respective white-light transmission spectra (shaded dashed lines). (c) Ensemble DsRed acceptor fluorescence lifetime $\tau_{\mathrm{A}}$ for different cavity resonance wavelengths. Blue and red arrows correspond to the two resonator configurations shown in (a) and (b).

A schematic diagram of the sample-microresonator system is shown in Fig. 3. While one of the resonator's mirrors is flat, the other is minimally curved with a radius of $R=150 \mathrm{~mm}$. This curvature is slight enough that the mirrors can still be assumed to be parallel, yet causes a well-defined variation in the mirror separation $L(x, y)$ in the resonator plane. The longitudinal

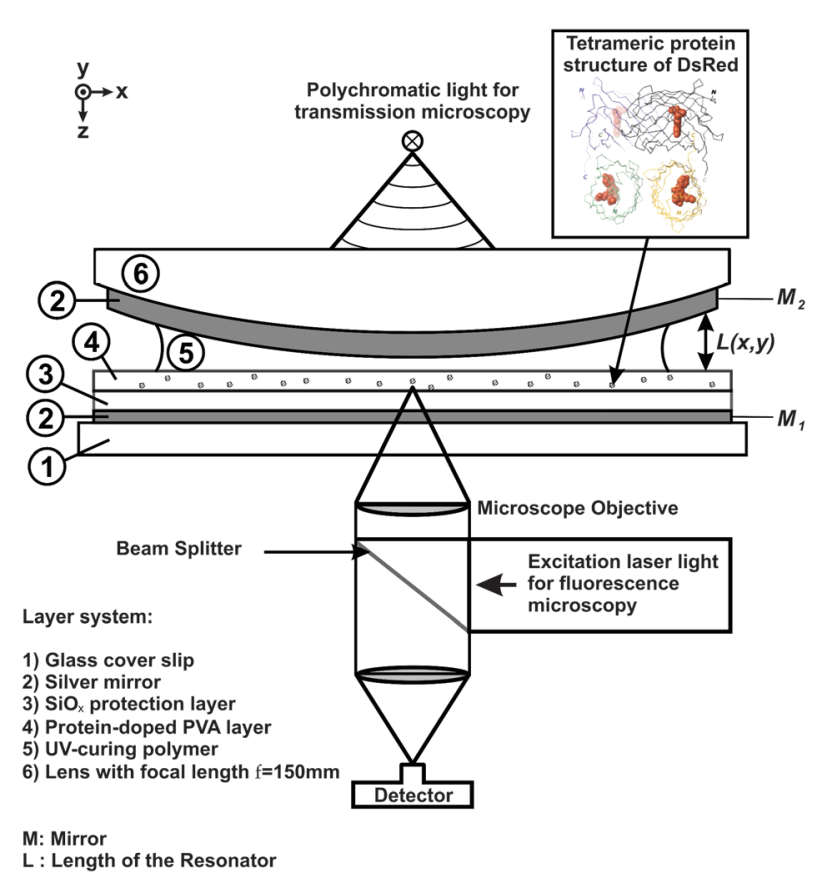

Fig. 3 Experimental setup consisting of a $\lambda / 2$ microresonator with embedded DsRed molecules in a physiological environment. The resonator is placed in a confocal laser microscope with an additional white-light source for measuring its transmission spectra. 
resonance wavelength can thus be tuned by scanning the detection point across the resonator.

The blue and red solid lines in Fig. 2(b) show the fluorescence spectra of DsRed in the microresonator for two different mirror separations $L$. The corresponding white-light transmission spectra, indicating the resonator's longitudinal resonances for the two mirror separations, are shown by the shaded dashed lines of the same color. The amplitudes of these spectra are not shown to scale but magnified to aid interpretation. Immediately, one can see that by choosing the correct resonance wavelength, one emission peak can be greatly enhanced while the other is nearly completely suppressed. For the blue curve, the normally dominant peak at $580 \mathrm{~nm}$ is so effectively suppressed by the resonator that it is visible only as a slight hump on the blue peak's flank. For the red curve, the off-resonance peak at $505 \mathrm{~nm}$ has completely disappeared. In both curves, the resulting peaks are asymmetric, showing a steep flank on the red side and a slow roll-off on the blue side. This is typical for emitters in a $\lambda / 2$-resonator as the longitudinal resonance wavelength also corresponds to the resonator's cutoff wavelength: light with a wavelength longer than the longitudinal resonance cannot populate any mode in the resonator. Shorter wavelengths, however, can populate off-axis modes which are no longer parallel to the $z$-axis but which can nevertheless be collected by the high NA of the used objective.

The spectra of single DsRed tetramers shown in Fig. 2(a) in blue (donor resonat) and red (acceptor resonat) illustrate that the influence of the resonator on transfer coupled systems is observable even on the single particle level. This enables a precise control and study of individual chromophores within one distinct transfer coupled complex, whose optical properties may vary by i.e. induced environmental influences. To verify that the influence of the resonator on the molecules' emission spectra is indeed an effect of their varied emission rates and not simply a filtering of the emitted light, the acceptor fluorescence lifetime $\tau_{\mathrm{A}}$ was studied as a function of the cavity resonance wavelength, viz. Fig. 2(c). The points show measured lifetimes and the curve is a calculation using the transfer matrix method (TMM) assuming a free-space fluorescence lifetime of $\tau_{\mathrm{rad}, \mathrm{fs}}^{\mathrm{A}}=6.7 \mathrm{~ns}$ and an emission quantum yield of $\Phi_{\mathrm{rad}, \mathrm{fs}}^{\mathrm{A}}=$ $25.2 \%$. The dramatic change in the measured lifetime agrees perfectly with the calculation's prediction. The red and blue arrows indicate the two resonator configurations at which the spectra in Fig. 2(b) were recorded, corresponding to the cases of inhibition (blue) and enhancement (red) of strong acceptor emission.

To study the resonator's effect quantitatively and to verify the rate-equation model presented above, we study the resonator-induced dequenching of the donor chromophore: when the resonator is tuned to the emission peak at $505 \mathrm{~nm}$, Fig. 2(b) shows that besides amplifying the donor emission, the acceptor fluorescence is effectively suppressed. If the quantum yield of the acceptor chromophore is sufficiently high, the lifetime of the $\mathrm{A}_{1}$ state will then be considerably increased. From eqn (1) one follows that an excited acceptor choromophore cannot participate in FRET and so this decay channel is lost to the donor. The resulting shift in the relative transition

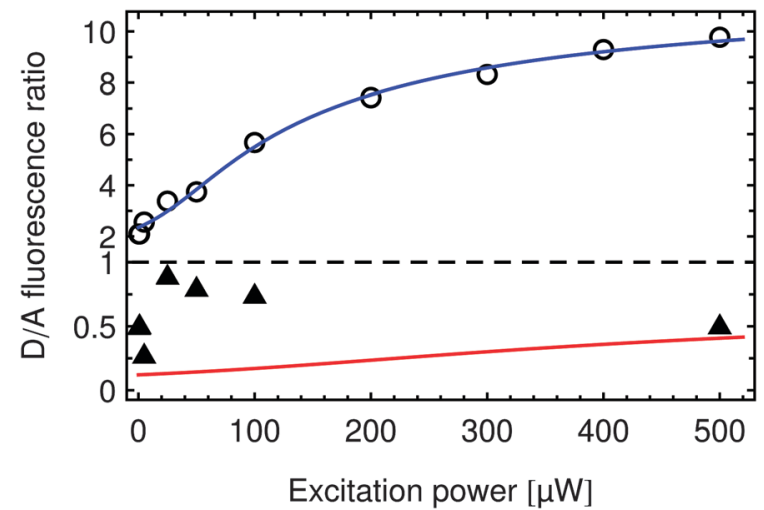

Fig. 4 Measured donor-to-acceptor fluorescence ratios of DsRed in free space (triangles) and in a microresonator tuned to the donor emission wavelength (circles) for varying illumination power $P_{\text {exc }}$. Blue line is the predicted behavior for the presented rate-equation model.

efficiency causes an increase in donor emission intensity compared to acceptor fluorescence. Fig. 4 shows the donor-to-acceptor fluorescence ratios for DsRed in a microresonator tuned to $505 \mathrm{~nm}$ (circles) and in free space (triangles), measured for increasing excitation power. In free space, this ratio remains in the order of 0.5 for all illumination power. With the acceptor fluorescence suppressed by the resonator, however, the donor dominates the fluorescence by a ratio of up to $10 / 1$ in the measured range.

Typically, this behavior is difficult to observe in free space, since, on the one hand, the fluorescence lifetime of a typical acceptor dye is rather short and, on the other hand, the fluorescence lifetime of a typical (unquenched) donor, being in the same range, is too long. Hence, the acceptor has already relaxed to the ground state while the donor is still excited, allowing for another energy transfer which quenches the emission of the donor. However, using a microresonator system, it is possible to precisely adjust the radiative rates of the respective chromophores. Thus, one can significantly shorten the lifetime of the donor chromophore while the lifetime of the acceptor chromophore is lengthened.

One might argue that the larger D/A fluorescence ratio in the resonator is simply due to the fact that the donor fluorescence is enhanced and the acceptor fluorescence is suppressed by the resonator, even without a change in the FRET efficiency. This static effect, however, should not depend on the illumination power $P_{\text {exc }}$. In fact, the effect of static fluorescence enhancement can be observed for $P_{\text {exc }} \rightarrow 0$. The modified fluorescence speed $k_{\text {rad }}$ thus causes a change from $f_{\mathrm{D}} / f_{\mathrm{A}} \approx 0.5$ to $f_{\mathrm{D}} / f_{\mathrm{A}} \approx 2.0$, while the illumination-dependent modification of the FRET efficiency increases the ratio to $f_{\mathrm{D}} / f_{\mathrm{A}} \approx 10$.

The dynamic behavior observed in the measurement is accurately reproduced by the rate equation model presented in this paper. The blue line in Fig. 4 shows the donor-toacceptor fluorescence ratio $D_{1} k_{\text {rad }}^{\mathrm{D}} /\left(A_{1} k_{\text {rad }}^{\mathrm{A}}\right)$ predicted by our model for decay efficiencies $\Phi_{x}=k_{x} / k_{\text {tot }}$ given in Table 1 . These values correspond to excited state fluorescence lifetimes of $2.8 \mathrm{~ns}$ and $2.6 \mathrm{~ns}$ for the uncoupled donor and acceptor, respectively, with fluorescence quantum yields (without FRET) of $18.1 \%$ and 
Table 1 Free-space decay efficiencies used in our model to reproduce the measured behavior

\begin{tabular}{lcll}
\hline Chromophore & $\Phi_{\text {rad,fs }}(\%)$ & $\Phi_{\mathrm{nr}, \mathrm{fs}}(\%)$ & $\Phi_{\mathrm{T}, \mathrm{fs}}(\%)$ \\
\hline Donor & 2.6 & 11.8 & 85.6 \\
Acceptor & 25.2 & 74.8 & - \\
\hline
\end{tabular}

$25.2 \%$. The FRET rate constant $k_{\mathrm{T}}$ then corresponds to a value of $2.1 \mathrm{GHz}$, in agreement with previously measured data. ${ }^{31}$ With these values, the model's predictions are in excellent agreement with our experimental results.

Having confirmed the accuracy of our model, we can now explore the parameter space of the studied system. In Fig. 5, we plot the donor-to-acceptor fluorescence ratio (red surface, left scale) and the FRET efficiency,

$$
\Phi_{\mathrm{T}}=\frac{k_{\mathrm{T}} A_{0}}{k_{\mathrm{T}} A_{0}+k_{\mathrm{rad}}^{\mathrm{D}}+k_{\mathrm{nr}}^{\mathrm{D}}},
$$

(green surface, right scale) for typical values of the resonator mirror separation $L$ and illumination power $P_{\text {exc }}$.

Many interesting features can be observed in this representation. First, one can see that the large increase in the D/A fluorescence ratio is only possible if the resonator is tuned to the correct wavelength. A large enhancement can be seen if the acceptor fluorescence is effectively inhibited while allowing, or even enhancing, donor emission. For larger $L$, both donor and acceptor emissions are allowed, and so the $\mathrm{D} / \mathrm{A}$ ratio is similar to that in free space ( $c f$. triangles in Fig. 4). For very small $L$, both donor and acceptor emissions are suppressed. While the $\mathrm{D} / \mathrm{A}$ ratio is not strongly enhanced in this case, it shows a saturation onset at very low power $P_{\text {exc }}$. This is due to the fact that, with fluorescence being inhibited, Förster transfer plays the dominant role in the energy dynamics of the coupled system. As the resonator modes prevent the acceptor from decaying radiatively, the resulting FRET inhibition is clearly visible already at very low power. Finally, one can see that the FRET efficiency $\Phi_{\mathrm{T}}$ varies greatly across the shown parameter space. Depending on the incident power, tuning the resonator mirrors allows us to reduce the FRET efficiency between 50\% and $75 \%$. It should be pointed out that this is not a modification of the FRET rate constant $k_{\mathrm{T}}$ as per the factor $F_{\mathrm{T}}$ (here, $F_{\mathrm{T}}=1$ ), as the resonator is not capable of sufficiently modifying the near

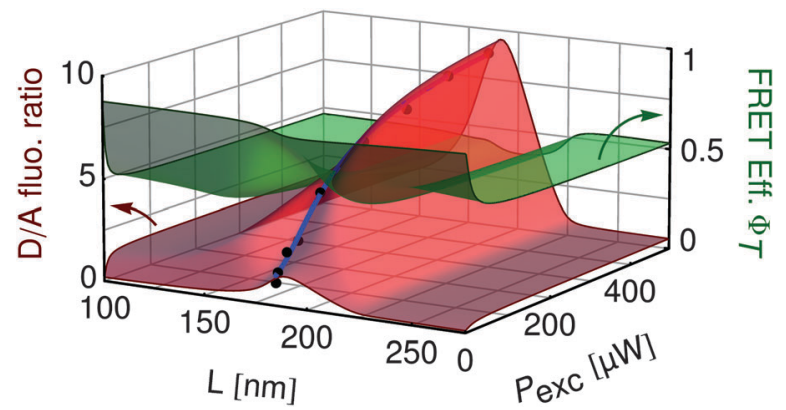

Fig. 5 Simulated D/A ratio (red surface) and FRET efficiencies (green surface) for different resonator widths $d$ and excitation power $P_{\text {exc. }}$. field of the donor dipole. Much more, it is an active modification of the other transition parameters $D_{1}$ and $A_{0}$, allowing us to selectively change the rate and efficiency of the Förster transfer.

\section{Acknowledgements}

Financial support from the German Research Council (DFG) for ME1600/13-1 is gratefully acknowledged.

\section{References}

1 T. Förster, Intermolecular energy migration and fluorescence, Ann. Phys., 1948, 2, 55-75.

2 T. A. Schüttrigkeit, U. Zachariae, T. von Feilitzsch, J. Wiehler, J. von Hummel, B. Steipe and M. E. Michel-Beyerle, Picosecond time-resolved fret in the fluorescent protein from discosoma red (wt-dsred), ChemPhysChem, 2001, 5, 325-328.

3 S. Brasselet, E. J. G. Peterman, A. Miyawaki and W. E. Moerner, Single-molecule fluorescence resonant energy transfer in calcium concentration dependent cameleon, J. Phys. Chem. B, 2000, 104, 3676-3682.

4 J. Hofkens, M. Cotlet, T. Vosch, P. Tinnefeld, K. D. Weston, C. Ego, A. Grimsdale, K. Müllen, D. Beljonne, J. Luc Brédas, S. Jordens, G. Schweitzer, M. Sauer and F. C. de Schryver, Revealing competitive Förster-type resonance energytransfer pathways in single bichromophoric molecules, Proc. Natl. Acad. Sci. U. S. A., 2003, 100(23), 13146-13151.

5 E. B. van Munster and T. W. Gadella, Fluorescence lifetime imaging microscopy (flim), Adv. Biochem. Eng./Biotechnol., 2005, 95, 143-175.

6 E. A. Jares-Erijman and T. M. Jovin, Fret imaging, Nat. Biotechnol., 2003, 21(11), 1387-1395.

7 A. A. Deniz, T. Laurence, G. S. Beligere, M. Dahan, A. B. Martin, D. S. Chemla, P. E. Dawson, P. G. Schultz and S. Weiss, Single molecule protein folding: diffusion Förster energy transfer studies of the denaturation of chymotrypsin inhibitor 2, Proc. Natl. Acad. Sci. U. S. A., 2000, 97, 5179-5184.

8 R. Gronheid, J. Hofkens, F. Köhn, T. Weil, E. Reuther, K. Müllen and F. C. de Schryver, Intramolecular forster energy transfer in dendritic systems at the single molecule level, J. Am. Chem. Soc., 2002, 124, 2418-2419.

9 J. R. Lakowicz, Principles of Fluorescence Spectroscopy, Kluwer Academic, New York, 2nd edn, 1999.

10 G. Baird, D. Zacharias and R. Tsien, Biochemistry, mutagenesis, and oligomerization of dsred, a red fluorescent protein from coral, Proc. Natl. Acad. Sci. U. S. A., 2000, 97(22), 11984-11989.

11 B. Lounis, J. Deich, F. I. Rosell, S. G. Boxer and W. E. Moerner, Photophysics of dsred, a red fluorescent protein, from the ensemble to the single-molecule level, J. Phys. Chem. B, 2001, 105, 5048-5054.

12 M. Matz, A. Fradkov, Y. Labas, A. Savitsky, A. Zaraisky, M. Markelov and S. Lukyanov, Fluorescent proteins from non-bioluminescent anthozoa species, Nat. Biotechnol., 1999, 17, 969-973. 
13 C. Blum, V. Subramaniam, F. Schleifenbaum, F. Stracke, B. Angres, A. Terskikh and A. J. Meixner, Single molecule fluorescence spectroscopy of mutants of the discosoma red fluorescent protein dsred, Chem. Phys. Lett., 2002, 362, 355-361.

14 F. Schleifenbaum, Energy transfer in the red fluorescent protein DsRed in confined optical fields, Life Sciences, Rhombos, Berlin, 2008, vol. 3.

15 M. Steiner, F. Schleifenbaum, C. Stupperich, A. V. Failla, A. Hartschuh and A. J. Meixner, Microcavity-controlled single-molecule fluorescence, ChemPhysChem, 2005, 6, 2190-2196.

16 M. Steiner, F. Schleifenbaum, C. Stupperich, A. V. Failla, A. Hartschuh and A. J. Meixner, A new microcavity design for single molecule detection, J. Lumin., 2006, 119(120), 167-172.

17 G. S. Agarwal and S. D. Gupta, Microcavity-induced modification of the dipole-dipole interaction, Phys. Rev. A: At., Mol., Opt. Phys., 1998, 57(1), 667-670.

18 M. J. A. Dood, J. Knoester, A. Tip and A. Polman, Förster transfer and the local optical density of states in erbium doped silica, Phys. Rev. B: Condens. Matter Mater. Phys., 2005, 71, 1151021.

19 S. D. Druger, S. Arnold and L. M. Folan, Theory of enhanced energy transfer between molecules embedded in spherical dielectric particles, J. Chem. Phys., 1987, 87(5), 2649-2659.

20 T. Kobayashi, Q. Zheng and T. Sekiguchi, Resonant dipole-dipole interaction in a cavity, Phys. Rev. A: At., Mol., Opt. Phys., 1995, 52(4), 2835-2846.

21 P. T. Leung and K. Young, Theory of enhanced energy transfer in an aerosol particle, J. Chem. Phys., 1988, 89(5), 2894-2899.
22 A. C. Pineda and D. Ronis, Classical model for energy transfer in microsperical droplets, Phys. Rev. E: Stat. Phys., Plasmas, Fluids, Relat. Interdiscip. Top., 1995, 52(5), 5178-5194.

23 P. Andrew and W. L. Barnes, Förster energy transfer in an optical microcavity, Science, 2000, 290, 785-788.

24 W. L. Barnes and P. Andrew, Energy transfer under control, Nature, 1999, 400, 505-506.

25 M. Hopmeier, W. Guss, M. Deussen, E. O. Göbel and R. F. Mahrt, Enhanced dipole-dipole interaction in a polymer microcavity, Phys. Rev. Lett., 1999, 82(20), 4118-4121.

26 A. Konrad, A. L. Trost, S. Skandary, M. Hussels, A. J. Meixner, N. V. Karapetyan and M. Brecht, Manipulating the excitation transfer in Photosystem I using a Fabry-Perot metal resonator with optical subwavelength dimensions, Phys. Chem. Chem. Phys., 2014, 16, 6175-6181.

27 G. Bjork, On the spontaneous lifetime change in an ideal planar microcavity-transition from a mode continuum to quantized modes, IEEE J. Quantum Electron., 1994, 30(10), 2314-2318.

28 A. Taflove and S. C. Hagness, Computational Electrodynamics: The Finite-Difference Time-Domain Method, Artech House, Boston, 2nd edn, 2000.

29 A. M. Kern and O. J. F. Martin, Surface integral formulation for 3D simulations of plasmonic and high permittivity nanostructures, J. Opt. Soc. Am. A, 2009, 26(4), 732-740.

30 C. Blum, N. Zijlstra, A. Lagendijk, M. Wubs, A. P. Mosk, V. Subramaniam and W. L. Vos, Nanophotonic control of the forster resonance energy transfer efficiency, Phys. Rev. Lett., 2012, 109(20), 203601.

31 F. Schleifenbaum, Energy transfer in the red fluorescent protein DsRed in confined optical fields, Phd thesis, 2008. 